\title{
Information transfer between respiration and heart rate during sleep apnea
}

\author{
Carolina Varon ${ }^{1,2}$, Luca Faes ${ }^{3}$, Dries Testelmans ${ }^{4}$, Bertien Buyse ${ }^{4}$, Sabine Van Huffel ${ }^{1,2}$ \\ ${ }^{1}$ KU Leuven, Department of Electrical Engineering-ESAT, STADIUS Center for Dynamical \\ Systems, Signal Processing and Data Analytics, Leuven, Belgium \\ ${ }^{2}$ iMinds Medical IT, Leuven, Belgium \\ ${ }^{3}$ BIOtech, University of Trento, and IRCS-PAT FBK, Trento, Italy \\ ${ }^{4}$ UZ Leuven, Department of Pneumology, Leuven, Belgium
}

\begin{abstract}
It is well-known that sleep apnea affects the respiration and the heart rate (HR), and studies have shown that the cardiorespiratory coupling is also compromised during obstructive sleep apnea (OSA). Furthermore, the classification of hypopneas is challenging, in particular when only ECG-derived features are used. In this context, this study investigates how different ECG-derived respiratory $(E D R)$ signals resemble the respiratory effort during different types of apneas, and how the amount of information transferred from respiration to HR varies according to the respiratory signal used, real or ECG-derived. ECG and respiratory signals of 10 patients suffering from sleep apnea were analysed, and three different EDR algorithms were used to estimate the respiratory effort. The information transfer was quantified using information dynamics on HR and both the real and estimated respiratory signals. Results suggest that the information transfer is reduced during all types of apneas/hypopneas, and they indicate that the EDR might not capture all variations in cardiorespiratory dynamics during hypopneas. As a result, the information transfer computed using the real respiratory signal achieve accuracies of up to $85 \%$ in the detection of sleep apnea with $76 \%$ of hypopneas correctly detected, compared to $79 \%$ achieved using the EDR with only $63 \%$ of correctly identified hypopneas.
\end{abstract}

\section{Introduction}

Sleep apnea is a sleep-related breathing disorder that is considered a risk factor for morbidity and mortality due to its long-term effect on the cardiovascular system [1]. Apneas and hypopneas are two types of respiratory events characterized, respectively, by a total absence or a reduction of airflow during at least 10 seconds [2]. Typically, sleep apnea is diagnosed using poly(somno)graphy (PSG), which is a sleep test associated with high costs and low comfort. Therefore, multiple studies have focused on the development of less intrusive and low cost systems for the detection of sleep apnea [1,3], which often include the ECG and an estimation of the respiratory effort, or socalled the ECG-derived respiration (EDR) [4-6].

Apneas are subdivided into obstructive (OSA), central (CEN), and mixed (MIX), and they all have an impact on the HR and the respiratory effort. During OSA the subject stops breathing despite the presence of respiratory effort, while during CEN the breathing stops but this time due to absence of respiratory effort. OSA and MIX combined are called MIX apneas. Hypopneas on the other hand, are characterized by a reduced airflow caused either by a partial obstruction (obstructive hypopnea: OSH), or by a lower respiratory effort (non-obstructive hypopnea: HPA), which results in a reduced oxygen saturation. Hypopneas are often not accompanied by any clear change in the respiratory effort nor in the HR. Consequently, they are the most challenging type of respiratory events to be detected using portable systems based on ECG and respiration [3].

It is well-known that obstructive apneas not only affect the HR and respiration, but also the cardiorespiratory coupling $[1,3]$ but little is known about how these interactions are affected during hypopneas, and if these can be used to improve the performance of detection algorithms. Hence, this work investigates the information transfer derived using information dynamics [7], between respiration and HR during different apneas and hypopneas. In addition, the impact of the respiratory signal used, real or estimated, on the information transfer is analysed. Finally, a classifier is used to assess the discrimination power of information transfer in the detection of sleep apnea.

\section{Methodology}

\subsection{Data}

The dataset used in this study consists of single-lead ECG and respiratory signals extracted from PSG record- 
ings of 10 patients of the University Hospitals Leuven. The mean age of the patients was $48.4 \pm 11.2$ years and their mean apnea-hypopnea index (AHI) was $33 \pm 20.9$. Two respiratory signals were acquired using respiratory belts around the chest and the abdomen, and in the remainder of this paper they will be denoted by $\mathrm{R}_{\mathrm{CH}}$ and $\mathrm{R}_{\mathrm{AB}}$, respectively. Both the ECG and the respiratory signals were sampled at $200 \mathrm{~Hz}$, and all the PSG recordings were annotated according to the Chicago Criteria [2]. These annotations indicate the beginning of the respiratory event, the duration, and the type: obstructive apnea (OSA); central apnea (CEN); obstructive hypopnea (OSH); non-obstructive hypopnea (HPA); or mixed apnea (MIX). In order to analyze the information transfer between respiration and heart rate during apnea events, the annotations were used to segment the signals into artefact-free epochs containing 30 seconds before the beginning of the events, the events, and $30 \mathrm{sec}-$ onds after the end of the events. In total, 2200 respiratory events were segmented, namely, 652 OSA, $1282 \mathrm{OSH}$, $134 \mathrm{HPA}, 97 \mathrm{CEN}$, and $35 \mathrm{MIX}$, with mean duration of $88.3 \pm 14.36$ seconds. Furthermore, segments of 1 minute containing normal (NOR) activity were selected and only 1417, which were free of artefacts were included in the study. All artefact-free segments were selected using the algorithm presented in [3].

\subsection{Data Processing}

In total, 3617 segments of ECG and respiratory signals were selected. From each ECG, the tachogram $(R R)$ was computed using the R-peak algorithm presented in [3], and three different EDR signals were derived. The first EDR was computed using the changes of the R-peak amplitude in a baseline corrected ECG signal, as first described in [4]. This EDR will be denoted by $R_{a m p}$. The second and third EDR signals were computed using principal component analysis (PCA) [5] and its kernel version (kPCA) [6], and they will be denoted by $R_{\text {pca }}$ and $R_{\text {kpca }}$, respectively. All tachograms and EDR signals were resampled at $5 \mathrm{~Hz}$ using cubic spline interpolation, and the real respiratory signals $\mathrm{R}_{\mathrm{CH}}$ and $\mathrm{R}_{\mathrm{AB}}$ were downsampled at the same frequency. After that, both types of respiratory signals, real and estimated, were high-pass filtered at $0.05 \mathrm{~Hz}$.

In order to evaluate how much the EDR signals resemble the real respiratory effort measured on the chest and abdomen, the correlation coefficient and the mean magnitude squared coherence (MSC) were computed between each pair of signals, as done in [6].

\subsection{Information Dynamics}

Information dynamics is a framework derived from the field of dynamical information theory, which allows to estimate the amount of information stored in a system (i.e. heart rate) and the information transferred from one system to the other (i.e. respiration to heart rate). In this work, these information measures were estimated using the time series method proposed in [7].

Assume a stationary stochastic process $\mathbf{U}=[\mathbf{X}, \mathbf{Y}]$, where $\mathbf{X}=\left\{X_{n}\right\}_{n=1}^{N}$ is the respiratory signal, and $\mathbf{Y}=$ $\left\{Y_{n}\right\}_{n=1}^{N}$ the tachogram, both of length $N$. These two systems are interconnected through the well-known respiratory sinus arrhythmia (RSA), which defines a causal relationship from $\mathbf{X}$ to $\mathbf{Y}$. Therefore, the uncertainty about $Y_{n}$ can be resolved by knowing its own past denoted as $\mathbf{Y}_{\mathbf{n}}^{-}=\left[Y_{n-1}, Y_{n-2, \ldots}\right]$ and the information transferred from the past of $\mathbf{X}$ denoted as $\mathbf{X}_{\mathbf{n}}^{-}=\left[X_{n-1}, X_{n-2}, \ldots\right]$. In fact, this uncertainty about $Y_{n}$ can be resolved by means of the predictive information $\left(P_{\mathbf{Y}}\right)$, defined as

$$
P_{\mathbf{Y}}=H\left(Y_{n}\right)-H\left(Y_{n} \mid \mathbf{X}_{\mathbf{n}}^{-}, \mathbf{Y}_{\mathbf{n}}^{-}\right),
$$

where the first term is the Shannon entropy, which quantifies the uncertainty contained in $Y_{n}$, and the second term is the conditional entropy, which quantifies the residual amount of information carried by $Y_{n}$ that cannot be predicted by the past of the process $\mathbf{U}$. In order to quantify the contribution to $Y_{n}$ of only $\mathbf{X}$, the definition in (1) can be rewritten as

$$
P_{\mathbf{Y}}=H\left(Y_{n}\right)-H\left(Y_{n} \mid \mathbf{Y}_{\mathbf{n}}^{-}\right)+H\left(Y_{n} \mid \mathbf{Y}_{\mathbf{n}}^{-}\right)-H\left(Y_{n} \mid \mathbf{X}_{\mathbf{n}}^{-}, \mathbf{Y}_{\mathbf{n}}^{-}\right),
$$

where $H\left(Y_{n}\right)-H\left(Y_{n} \mid \mathbf{Y}_{\mathbf{n}}^{-}\right)$is the self-entropy denoted by $S_{\mathbf{Y}}$, and $H\left(Y_{n} \mid \mathbf{Y}_{\mathbf{n}}^{-}\right)-H\left(Y_{n} \mid \mathbf{X}_{\mathbf{n}}^{-}, \mathbf{Y}_{\mathbf{n}}^{-}\right)$is the transfer entropy denoted by $T_{X \rightarrow Y}$. Here, $S_{\mathbf{Y}}$ is the amount of information stored in $\mathbf{Y}$, where larger values indicate a high predictability of the heart rate. On the other hand, $T_{X \rightarrow Y}$ quantifies the amount of information transferred from the respiration to the heart rate, which cannot be predicted from the past of the heart rate. Hence, differences in cardiorespiratory interactions can be found by analysing this term. The larger the transfer entropy, the more the information transferred from respiration to heart rate.

The different entropy terms can be computed using the approach presented in [7], which links information theory and predictability. This approach assumes that the process $\mathbf{U}$ has a joint Gaussian distribution that allows to describe its dynamics using a linear vector autoregressive (VAR) model of order $p$, optimized using the Akaike information criterion (AIC). In this way, the different entropy terms are linked to the error probabilities of a regression model.

\subsection{Comparative analysis}

The differences between the entropy estimates of different apnea types (OSA, OSH, HPA, CEN, and MIX) and normal segments were evaluated using the Kruskal-Wallis test with 95\% of confidence. Since 6 different groups (i.e. 5 apnea types and 1 normal group) were compared all versus all, a multi-comparison test was used with Bonferroni 
correction equal to $\alpha / 15=0.003$, with $\alpha=0.05$, and 15 hypothesis. These differences were analysed for each respiratory signal: 2 real and 3 estimated. Furthermore, differences in the values of coherence and correlation between the EDRs and the real respiratory signals were evaluated for each type of apnea using the same statistical tests.

In addition to the statistical tests, a classifier was used to assess the discriminative power of the transfer entropy in the detection of sleep apnea. A least-squares support vector machine (LS-SVM) classifier was used as in [3], with the following input parameters: standard deviation of $R R$ $(\operatorname{std}(R R))$ and $T_{X \rightarrow Y}$. The latter was calculated using the real and estimated respiratory signals. In total, 5 different classifiers were implemented and a comparison was performed using their sensitivity, specificity, and accuracy.

\section{Results and discussion}

The comparison between the entropy estimates indicates that the information transferred from respiration to heart rate is significantly lower $(\mathrm{p}<0.003)$ during all apnea episodes than during normal activity. This result can be seen in Figure 1, where the transfer entropy values computed using both the abdominal $\left(\mathrm{R}_{\mathrm{AB}}\right)$ and thoracic $\left(\mathrm{R}_{\mathrm{CH}}\right)$ respiratory effort are significantly lower for all apneas. This can be explained by the increased sympathetic modulation during episodes of apnea, which cause changes in heart rate that are not modulated by the respiration. In other words, the synchronization between respiration and heart rate (i.e. RSA) is reduced during episodes of sleep apnea [1]. These results are in agreement with those presented in [3], where the cardiorespiratory interactions were quantified using orthogonal subspace projections, and where the power of the modulations of heart rate due to respiration was found to be significantly reduced during apneas. An important difference between this work and the one described in [3] is that here the hypopneas are also characterized by a reduced information transfer, while in [3] they appeared similar to normal activity. Note, however, that this is the case only when the real respiratory signal is used. As shown in Figure 1, the difference between hypopneas and normal activity is not captured by any of the EDR approaches: $R_{\text {amp }}, R_{\text {pca }}$ and $R_{\text {kpca }}$. This can be related to the reduced correlation and coherence between the EDRs and the respiratory effort during apnea episodes, which was found to be significant $(\mathrm{p}<0.003)$ for all the EDRs. These results are depicted in Figure 2. Note that this reduction is more pronounced in OSA, OSH, CEN, and MIX apneas, where the respiratory effort is known to differ significantly from normal activity [2]. Furthermore, even though the multi-comparison test indicates a significant difference, it is noticeable that the relationship between the real respiratory effort and the EDR during hypopneas (HPA) does not seem to differ from the one during normal activity. This is not a surprise, since the detection of hypopneas requires the use of other physiological information apart from respiratory effort, such as blood oxygen saturation and airflow. With this in mind, it is possible that the small variations that might be present in the respiratory effort during hypopneas are not captured by the EDR signal. In addition, the large information transfer during NOR and HPA can be associated, on the one hand, with the fact that both the EDR and the tachogram are surrogate signals of the ECG, and on the other hand with the minimal effect that hypopneas have on the morphology of the ECG [3]. Further investigations are needed to determine if this is the case. Moreover, since the model used in this study assumes stationarity and a join Gaussian distribution between the variables, it is important to validate these results against model free approaches.

From the results of transfer entropy obtained using the real respiratory effort, it is possible to think that an improved detection of hypopneas can be achieved. In order to test this, 5 classifiers were built and their sensitivity (Sens), specificity (Spec), accuracy (Acc.), and area under the receiver operating curve (AUC) were compared and they are indicated in Table 1. The table also contains the percentage of HPA that were classified correctly in each case. Note that the classification performance obtained with the real respiratory signals is better than the one obtained using any of the EDRs. At this point only two features were used for the classification, namely, the standard deviation of the tachogram and $T_{X \rightarrow Y}$. Future studies will focus on the use of extra parameters that can help improving the overall classification performance on bigger datasets.

\section{Conclusion}

The findings presented in this study suggest that the cardiorespiratory interactions are affected during all apneas. In fact, the information transferred from respiration to heart rate is reduced during episodes of apnea, which confirms the lower synchronization suggested by previous studies in the literature. These findings, however, were only observed during hypopneas when the real respiratory effort was used to quantify the information transfer. One possible explanation for this is the fact that the EDR does not fully capture the respiratory variations during apneas. As a result, the use of the EDR might reduce the performance of sleep apnea detectors, in particular, in the detection of hypopneas. Note that this work was done using predefined segments containing the apnea episodes, hence, future studies need to be performed on continuous signals in order to confirm the discrimination power of the transfer entropy in combination with the EDR. Furthermore, methods that do not assume stationarity and joint Gaussian distributions will need to be used to estimate the information transfer in order to validate these results. 

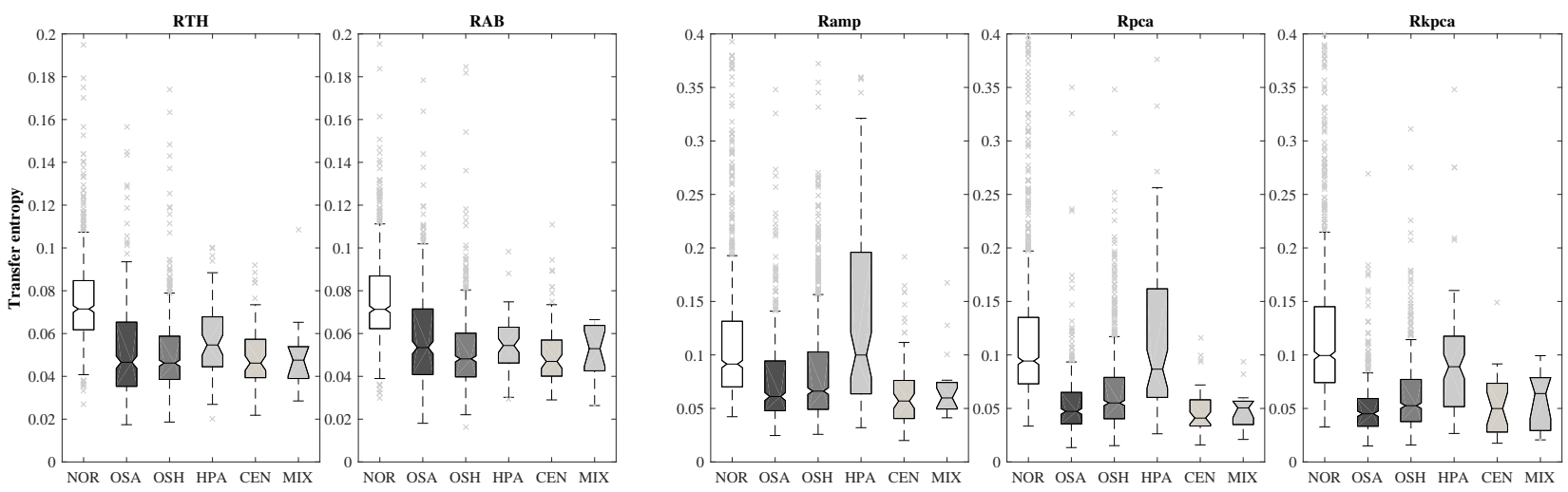

Figure 1. Transfer entropy for normal segments and the different apneas and hypopneas. Note that the distinction between hypopneas and normal activity disappears when the EDR signals are used.

Table 1. Performance of the classifiers using $T_{X \rightarrow Y}$ and $\operatorname{std}(R R)$. \%HPA corresponds to the percentage of hypopneas that are correctly classified.

\begin{tabular}{|c|c|c|c|c|c|}
\hline Resp. Signal & Sens. & Spec. & Acc. & AUC & $\%$ HPA \\
\hline $\mathrm{R}_{\mathrm{AB}}$ & 0.81 & 0.86 & 0.83 & 0.89 & 0.75 \\
$\mathrm{R}_{\mathrm{CH}}$ & 0.88 & 0.81 & $\mathbf{0 . 8 5}$ & 0.89 & $\mathbf{0 . 7 6}$ \\
$\mathrm{R}_{\text {amp }}$ & 0.77 & 0.83 & $\mathbf{0 . 7 9}$ & 0.85 & $\mathbf{0 . 6 3}$ \\
$\mathrm{R}_{\text {pca }}$ & 0.75 & 0.83 & 0.79 & 0.85 & 0.60 \\
$\mathrm{R}_{\text {kpca }}$ & 0.75 & 0.85 & 0.81 & 0.84 & 0.54 \\
\hline
\end{tabular}
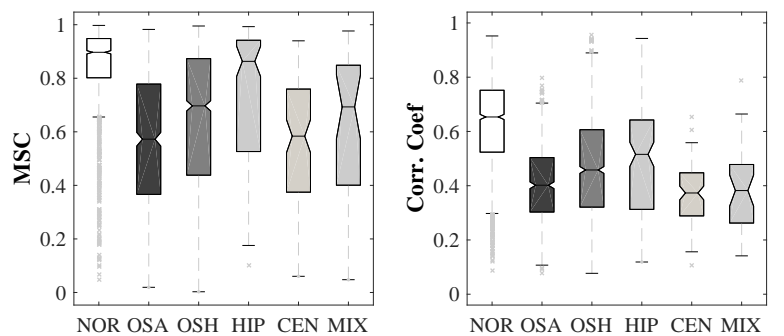

Figure 2. Mean magnitude squared coherence (MSC) and correlation coefficient between $\mathrm{R}_{\mathrm{AB}}$ and $\mathrm{R}_{\mathrm{amp}}$. The results are similar for all EDRs and for $\mathrm{R}_{\mathrm{CH}}$.

\section{Acknowledgements}

Bijzonder Onderzoeksfonds KU Leuven (BOF): Center of Excellence (CoE) \#: PFV/10/002 (OPTEC), SPARKLE Sensor-based Platform for the Accurate and Remote monitoring of Kinematics Linked to E-health \#: IDO-13-0358, The effect of perinatal stress on the later outcome in preterm babies \#: C24/15/036. Fonds voor Wetenschappelijk OnderzoekVlaanderen (FWO): G.0869.12N (Tumor imaging), G.0A5513N (Deep brain stimulation). Agentschap voor Innovatie door Wetenschap en Technologie (IWT): TBM 110697 - NeoGuard, STW 150466 - OSA+. iMinds Medical Information Technologies: Dotatie-Strategisch basis onderzoek (SBO- 2016). Belgian Federal Science Policy Office: IUAP \#P7/19/ (DYSCO, 'Dynamical systems, control and optimization', 2012-2017). Belgian Foreign Affairs-Development Cooperation: VLIR UOS programs (2013-2019). EU: European Union's Seventh Framework Programme (FP7/2007-2013): EU MC ITN TRANSACT 2012, \#316679,
ERASMUS EQR: Community service engineer , \#539642-LLP-1-2013. Other EU: INTERREG IVB NWE programme \#RECAP 209G. European Research Council: The research leading to these results has received funding from the European Research Council under the European Union's Seventh Framework Programme (FP7/2007-2013) / ERC Advanced Grant: BIOTENSORS (n 339804). This paper reflects only the authors' views and the Union is not liable for any use that may be made of the contained information.

\section{References}

[1] Penzel $\mathrm{T}$ et al . Cardiovascular and respiratory dynamics during normal and pathological sleep. Chaos Mar 2007; 17(1):015116.

[2] Task Force AASM . Sleep-related breathing disorders in adults: recommendations for syndrome definition and measurement techniques in clinical research. Sleep Aug 1999; 22(5):667-689.

[3] Varon $\mathrm{C}$ et al . A novel algorithm for the automatic detection of sleep apnea from single-lead ECG. IEEE Transactions on Biomedical Engineering Sep 2015;62(9):2269-2278.

[4] Moody GB et al . Derivation of respiratory signals from multi-lead ecgs. Proc Computers in cardiology 1985; 12(1985):113-116.

[5] Langley P et al . Principal component analysis as a tool for analyzing beat-to-beat changes in ecg features: application to ecg-derived respiration. IEEE Trans Biomed Eng Apr 2010; 57(4):821-829.

[6] Widjaja D et al . Application of kernel principal component analysis for single-lead-ecg-derived respiration. IEEE Trans Biomed Eng Apr 2012;59(4):1169-1176.

[7] Faes L et al . Information decomposition in bivariate systems: theory and application to cardiorespiratory dynamics. Entropy 2015;17(1):277-303.

Address for correspondence:

Carolina Varon

ESAT/STADIUS/KU Leuven

Kasteelpark Arenberg 10, bus 2446, 3001 Leuven, Belgium. carolina.varon@esat.kuleuven.be 